Xiaodong Cao*, Mengwei Yu, Georg Bauer und Christian Rembe

\title{
Bestimmung des Anregungsspektrums für kontaktlose breitbandige Messungen der Übertragungsfunktion mit Plasmaanregung und Laser-Doppler-Vibrometrie
}

Determination of the excitation spectrum for contactless broadband measurements of the frequency response function with plasma excitation and laser Doppler vibrometry

\section{DOI 10.1515/teme-2020-0015}

Zusammenfassung: Die Übertragungsfunktion eines mechanischen Systems ist eine der wichtigsten Messgrößen in der Schwingungsanalyse. Um die Übertragungsfunktion möglichst breitbandig bestimmen zu können, wird eine breitbandige Anregung benötigt, welche z.B. durch eine Plasmaanregung realisiert werden kann. Allerdings stellt die Bestimmung des Spektrums einer solchen Plasmaanregung, welche für die Ermittlung der Übertragungsfunktion notwendig ist, zurzeit noch eine Herausforderung in der Forschung dar. In diesem Beitrag bestimmen wir das Anregungsspektrum durch ein Simulationsmodell und vergleichen das Ergebnis mit dem Resultat einer bisher typischerweise eingesetzten experimentellen Schätzmethode. Der vorgestellte Ansatz benötigt einen deutlich geringeren Aufwand und stellt damit für praktische Anwendungen eine gute Alternative zum aktuellen Stand der Technik dar.

Schlüsselwörter: Plasmaanregung, FRF, LDV.

Abstract: The frequency response function (FRF) of a mechanical system is one of the most important measured characteristics in vibration analysis. To determine the FRF as broadband as possible, a broadband excitation, such as plasma excitation, is required. However, determination of the spectrum of the plasma excitation, which is necessary for the determination of FRF, is still a challenge to research. In this paper we determine the excitation

\footnotetext{
*Korrespondenzautor: Xiaodong Cao, Institut für Elektrische Informationstechnik, TU Clausthal, Leibnizstraße 28, 38678 Clausthal-Zellerfeld, Deutschland, E-Mail:

xiaodong.cao@tu-clausthal.de

Mengwei Yu, Georg Bauer, Christian Rembe, Institut für Elektrische Informationstechnik, TU Clausthal, Leibnizstraße 28, 38678 Clausthal-Zellerfeld, Deutschland, E-Mail: mengwei.yu@tuclausthal.de, bauer@iei.tu-clausthal.de rembe@iei.tu-clausthal.de
}

spectrum by a simulation model and compare the result with the outcome of a typical experimental approach. The presented method requires significantly less effort and is therefore a good alternative to the state of the art for practical applications.

Keywords: Laser-induced excitation, FRF, LDV.

\section{Einleitung}

Die Übertragungsfunktion (Frequency-Response-Function - FRF) eines mechanischen Systems zählt zu den wichtigsten Messgrößen in der Schwingungsanalyse. Klassisch werden FRF-Messungen oft im Frequenzbereich bis zu einigen Kilohertz durchgeführt. Allerdings ist der Bedarf an breitbandigen Schwingungsanalysen in den letzten Jahren sehr gestiegen. Dabei ist es ein Ziel, dynamische Eigenschaften für ein deutlich breiteres Spektrum als bisher bestimmen zu können, d.h. im Bereich von mehreren zehn Kilohertz bis etwa 100 Kilohertz. Im Bereich der vibrationsbasierten Schadenserkennung [1-3] werden z.B. breitbandige Schwingungsmessungen benötigt, um die Sensitivität bei der Schadenserkennung zu verbessern, weil Schädigungen an Bauteilen bei Resonanzfrequenzen im höheren Frequenzbereich eine stärkere Veränderung erwirken. LaserDoppler-Vibrometer können problemlos Schwingungen mit so großen Schwingungsbandbreiten messen (Bandbreiten bis in den Gigahertz-Bereich sind möglich [9]). Allerdings muss für eine breitbandige FRF-Messung bis z.B. $100 \mathrm{kHz}$ auch die Anregungskraft entsprechend breitbandig erzeugt und ermittelt werden können. Dies ist zurzeit noch eine Herausforderung für die Forschung.

Mit den konventionellen Anregungstechniken, wie z.B. Modalhammer und Modalshaker, werden bis auf spezielle Ausnahmen Frequenzen bis $10 \mathrm{kHz}$ angeregt [8]. In [6] wird eine FRF-Messmethode mit Hilfe von Laserablation 
vorgestellt. Die durch Laserablation erzeugte Anregung ist impulsartig, wobei die Impulsdauer deutlich kürzer als die von einem Modalhammerschlag ist. Daher ist das Anregungsspektrum wesentlich breitbandiger als es konventionelle Techniken ermöglichen. Das Anregungsspektrum der Laserablation wird dabei durch die Beschleunigung der Masse eines Pendels geschätzt. Um das Anregungsspektrum möglichst genau schätzen zu können, muss ein Pendelsystem für den zu betrachtenden Frequenzbereich entsprechend ausgelegt werden (siehe Abschnitt 2.1). Außerdem können parasitäre Einflüsse auf das Pendelsystem $\mathrm{zu}$ Messabweichungen führen.

In diesem Beitrag verwenden wir die von einem laserinduzierten Plasma erzeugte Stoßwelle als breitbandige kontaktlose Anregungsquelle. Wir schätzen das Anregungsspektrum direkt mit einem neuen Simulationsmodell und vergleichen das Ergebnis mit der Kraftschätzung durch die Pendelmethode. Bei der Pendelmethode muss vor jeder Messung mit einer Parameteränderung, beispielsweise der Änderung des Abstandes zwischen dem Plasma und dem Testobjekt, der Impuls vermessen werden. Die Schätzmethode mit Hilfe des Simulationsmodells benötigt keine Zusatzmessung und ist daher gut geeignet für praktische Anwendungen.

\section{Methode}

\subsection{Kraftschätzung mit einem Pendel}

Eine Methode zur Schätzung des breitbandigen Anregungskraftspektrums durch das laserinduzierte Plasma wurde in $[6,7]$ vorgestellt. Dabei wird vor der eigentlichen Messung ein Versuch an einem Pendel durchgeführt. Der Körper der Pendelmasse muss so ausgelegt sein, dass seine erste Eigenfrequenz mindestens das Doppelte der oberen Grenzfrequenz des betrachteten Frequenzbereichs beträgt [6]. In diesem Fall kann die Pendelmasse mit dem Gewicht $m$ als Starrkörper betrachtet werden. Für sehr kleine Pendelbewegungen kann das Pendelsystem vereinfacht als ein ungedämpfter Einmassenschwinger betrachtet werden. Aus der Bewegungsgleichung lässt sich die Übertragungsfunktion des ungedämpften Einmassenschwingers in der Form [4]

$$
\frac{\underline{A}(\omega)}{\underline{F}(\omega)}=\frac{\omega^{2}}{\omega^{2} m-k}
$$

herleiten. Dabei bezeichnen $\underline{A}(\omega)$ und $\underline{F}(\omega)$ die Fouriertransformierten des Beschleunigungs- und Kraftverlaufs. Die Parameter $\omega$ und $k$ sind die Kreisfrequenz und die Federsteifigkeit des Einmassenschwingersystems. Aus Gl. (1) ist ersichtlich, dass für $\omega \gg \omega_{0}$ mit der Eigenfrequenz $\omega_{0}=\sqrt{\frac{k}{m}}$ gilt:

$$
\omega^{2} m \gg k \Rightarrow \frac{\underline{A}(\omega)}{\underline{F}(\omega)} \approx \frac{1}{m} \text { bzw. } \underline{F}(\omega) \approx m \underline{A}(\omega) .
$$

Gl. (2) ist die fundamentale Idee dieser Schätzmethode. D.h. wenn der zu betrachtende Frequenzbereich mit großem Abstand zwischen der Eigenfrequenz des Pendelsystems $\omega_{0}$ und der ersten Eigenfrequenz des Körpers der Pendelmasse $\omega_{\mathrm{P}}$ liegt, ist das Kraftspektrum mit dem Faktor $m$ proportional zum Beschleunigungsspektrum. Mit der Fadenlänge des Pendels $l$ und der Erdbeschleunigung $g$ lässt sich die Eigenfrequenz des Pendelsystems $\omega_{0}=\sqrt{\frac{g}{l}}$ berechnen. Die Eigenfrequenz $\omega_{\mathrm{P}}$ kann z.B. mit Hilfe eines Finite-Elemente-Programms abgeschätzt werden. Somit kann das Kraftspektrum aus dem gemessenen Beschleunigungsspektrum bei bekannter Pendelmasse $m$ für den Frequenzbereich von mindestens $2 \omega_{0}$ bis maximal $\frac{\omega_{P}}{2}$ berechnet werden.

\subsection{Schätzung nach Simulationsmodell}

Die von einem laserinduzierten Plasma in einer idealen gleichmäßigen Atmosphäre erzeugte Stoßwelle kann vereinfacht als sphärische Welle betrachtet werden. Der Wellenfrontdruck der Stoßwelle lässt sich analytisch mit einem physikalischen Modell näherungsweise beschreiben [5]. Nach [10] kann der Druckverlauf einer starken sphärisch divergierenden Luftstoßwelle an der Stelle $r$ zum Zeitpunkt $t$ durch die Gleichung

$$
p_{\mathrm{st}}(r, t)=p_{\mathrm{st}}(r) H\left(t-t_{0, \mathrm{st}}(r)\right) e^{\left[-\left(t-t_{0, \mathrm{st}}(r)\right)\left(\varepsilon t_{0, \mathrm{st}}(r)\right)^{-1}\right]}
$$

annähernd beschrieben werden. Dabei ist $H(t)$ die Heaviside-Schrittfunktion, $\varepsilon=0,5$ eine Konstante und $r$ die Entfernung zwischen der Wellenfront und dem Druckursprung. Die räumliche Druckverteilung der Stoßwellenfront $p_{\text {st }}(r)$ lässt sich wie folgt berechnen:

$$
p_{\text {st }}(r)=\left(\frac{2}{5}\right)^{2} \frac{2 \xi_{0}^{5}}{\gamma+1} E r^{-3} .
$$

Dabei ist $\gamma=1,23$ der Isentropenexponent der Luft und $E$ die freigesetzte Energie. Der dimensionslose Parameter $\xi_{0}$ ist die Ähnlichkeitsvariable [12] der selbstähnlichen Wellenbewegung an der Wellenfront [14]. Dieser Parameter ist von $\gamma$ abhängig und beträgt in diesem Fall aus der Energieerhaltung den Wert von $\xi_{0} \approx 0,93$ [13, 14]. Aus der räumlichen Geschwindigkeitsverteilung der Stoßwel- 
lenfront [14]

$$
v_{\text {st }}(r)=\frac{2}{5} \xi_{0} \frac{5}{2}\left(\frac{E}{\rho_{0}}\right)^{\frac{1}{2}} r^{-\frac{3}{2}}
$$

mit der Dichte der Luft $\rho_{0}=1,205 \frac{\mathrm{kg}}{\mathrm{m}^{3}}$ wird der Zeitpunkt für die Ankunft der Wellenfront an der Stelle $r$

$$
t_{0, \mathrm{st}}(r)=\int_{0}^{r} \frac{\mathrm{d} r^{\prime}}{v_{\mathrm{st}\left(r^{\prime}\right)}}=\left(\frac{r}{\xi_{0}}\right)^{\frac{5}{2}}\left(\frac{\rho_{0}}{E}\right)^{\frac{1}{2}}
$$

berechnet. Der Druck wird bis auf einen Wert abfallen, der kleiner als der atmosphärische Druck ist. Dabei treten in der Druckverteilung positive und negative Phasen auf. Daher wird zu Gl. (3) ein exponentieller Term hinzugefügt, um die Unterdruckphase darzustellen [5]:

$$
\begin{aligned}
& p_{\mathrm{st}}(r, t)= \\
& p_{\mathrm{st}}(r)\left\{H\left(t-t_{0, \mathrm{st}}(r)\right)\left[1,2 e^{\frac{t_{0, \mathrm{st}}(r)-t}{\varepsilon t_{0, \mathrm{st}}(r)}}-0,2\right]\right. \\
& \left.-H\left(t-3,5 t_{0, \mathrm{st}}(r)\right)\left[1,2 e^{0,36 \frac{t_{0, \mathrm{st}}(r)-t}{\varepsilon t_{0, \mathrm{st}}(r)}}-0,2\right]\right\} .
\end{aligned}
$$

Die Geschwindigkeit der Stoßwellenfront sinkt mit steigendem Abstand $r$ kontinuierlich bis zur Schallgeschwindigkeit $v_{0}=343 \frac{\mathrm{m}}{\mathrm{s}}$ an der Stelle $r=r_{0}$. Mit Gl. (5) lässt sich $r_{0}$ mit $v_{\text {st }}\left(r_{0}\right)=v_{0}$ berechnen. Ab dieser Stelle breitet sich die Stoßwelle mit der Schallgeschwindigkeit $v_{0}$ weiter aus [10]. Daher werden der Druck und die Bewegung der Wellenfront für $r>r_{0}$ anhand der Ausbreitungseigenschaften einer sphärischen Schallwellen geschätzt:

$$
p_{\mathrm{ak}}(r)=\frac{C}{r} .
$$

Der Parameter $C$ ist hier eine Konstante, die die proportionale Beziehung zwischen Druck und Abstand beschreibt. Der Anfangsdruck einer sphärischen Schallwelle kann durch den Druck der Stoßwellenfront an der Position $r_{0}$ bestimmt werden:

$$
\begin{aligned}
p_{\mathrm{ak}}\left(r_{0}\right)=p_{\mathrm{st}}\left(r_{0}\right) & =\frac{C}{r_{0}}=\left(\frac{2}{5}\right)^{2} \frac{2 \xi_{0}^{5}}{\gamma+1} E r_{0}^{-3} \\
\operatorname{mit} C & =\left(\frac{2}{5}\right)^{2} \frac{2 \xi_{0}^{5}}{\gamma+1} E r_{0}^{-2} .
\end{aligned}
$$

Somit beträgt der Wellenfrontdruck im späteren Ausbreitungsstadium

$$
p_{\mathrm{ak}}(r)=\left(\frac{2}{5}\right)^{2} \frac{2 \xi_{0}^{5}}{\gamma+1} E r_{0}^{-2} r^{-1}
$$

Mit Bezug auf Gl. (7) kann der Wellenfrontdruck nach der Stelle $r_{0}$ durch die Gleichung

$$
\begin{aligned}
& p_{\mathrm{ak}}(r, t)= \\
& p_{\mathrm{ak}}(r)\left\{H\left(t-t_{0, \mathrm{ak}}(r)\right)\left[1,2 e^{\frac{t_{0, \mathrm{ak}}(r)-t}{\varepsilon t_{0, \mathrm{st}}(r)}}-0,2\right]\right. \\
& -H\left(t-t_{0, \mathrm{ak}}(r)-2,5 t_{0, \mathrm{st}}\left(r_{0}\right)\right) \cdot \\
& \left.\cdot\left[1,2 e^{0,36 \frac{t_{0, \mathrm{ak}}(r)-t}{\varepsilon t_{0, \mathrm{st}}\left(r_{0}\right)}}-0,2\right]\right\}
\end{aligned}
$$

mit

$$
t_{0, \mathrm{ak}}(r)=t_{0, \mathrm{st}}\left(r_{0}\right)+\frac{r-r_{0}}{v_{0}}
$$

angenähert beschrieben werden. Während der Interaktion zwischen der Stoßwelle und der Oberfläche des Testobjekts finden äußerst komplexe Reflexions- und Brechungsprozesse statt. Um die Wechselwirkung der Stoßwelle mit der Oberfläche des Testobjekts abschätzen zu können, wird ein lineares Schallwellenreflexionsmodell [5] zur Beschreibung der Stoßwellenreflexion angenommen. Die potentielle Energie durch den Druck einer Schallwelle ist umgekehrt proportional zur Dichte. Da die Dichte im Messobjekt um ein Vielfaches höher als in der Luft ist, wird nur ein geringer Teil der Energie in das Medium eingekoppelt, so dass hier die Druckbedingung für die schallharte Wand angenommen wird. Demnach sollte der Stoßwellendruck auf der Oberfläche des Testobjekts wegen der vollständigen Reflexion doppelt so hoch sein wie der einfallende Wellendruck $[5,10]$. Daher wird der Normaldruck $p_{n}(r, t)$ an der Testobjektoberfläche durch die Gleichung

$$
p_{n}(r, t)=2 p(r, t)
$$

mit

$$
\begin{aligned}
p(r, t) & =p_{\mathrm{st}}(r, t) \text { für } r \leq r_{0} \\
\text { und } p(r, t) & =p_{\mathrm{ak}}(r, t) \text { für } r>r_{0}
\end{aligned}
$$

beschrieben. Es wird außerdem angenommen, dass die Stoßwellenfront nur Kräfte an der Oberfläche erzeugt, die senkrecht auf die Oberfläche wirken.

Um die gesamte Anregungskraft auf einer rechteckigen Fläche zu berechnen, wird die Fläche durch ein feines äquidistantes Gitter mit Gitterabstand $\Delta b$ abgebildet. Der Druck an jedem dieser Gitterknoten lässt sich mit Gl. (13) berechnen und wird als konstant auf einem Flächenelement der Größe $\Delta b^{2}$ angenommen. Die gesamte Anregungskraft auf der Fläche $F_{\text {ges }}(t)$ zum Zeitpunkt $t$ ist somit näherungsweise die Summe aller Anregungskräfte $F_{n, i}\left(r_{i}, t\right)$, die jeweils auf das entsprechende Flächenelement $i$ wirken, 
d.h.

$$
\begin{aligned}
F_{\text {ges }}(t) & =\sum_{i=1}^{N} F_{n, i}\left(r_{i}, t\right) \\
& =\sum_{i=1}^{N} p_{n, i}\left(r_{i}, t\right) \Delta b^{2}
\end{aligned}
$$

Hier bezeichnet $N$ die Anzahl der Flächenelemente des Gitters und $r_{i}$ den Abstand zwischen dem Druckursprung und dem Mittelpunkt des Flächenelementes $i$. Der Gitterabstand $\Delta b$ wurde dabei so fein gewählt, dass eine weitere Reduzierung von $\Delta b$ keine signifikante Änderung bewirkt.

\subsection{Versuchsaufbau zur Kraftschätzung durch Pendelmethode}

Um das Ergebnis der Schätzung nach dem entwickelten Simulationsmodell zu validieren, wird ein Pendelsystem unter Berücksichtigung der in Abschnitt 2.1 genannten Randbedingungen ausgelegt. Ein Würfelblock aus Aluminium mit einer Kantenlänge von $20 \mathrm{~mm}$ wird als Pendelmasse eingesetzt. Das Gewicht der Pendelmasse beträgt $0,02216 \mathrm{~kg}$. Eine erste Untersuchung mit Hilfe des Finite-Elemente-Programms Ansys zeigt, dass die erste Eigenfrequenz des Körpers der Pendelmasse bei ungefähr $\omega_{\mathrm{P}}=70 \mathrm{kHz}$ liegt. Dies erlaubt eine Schätzung des Anregungsspektrums bis zu Frequenzen von $\frac{70 \mathrm{kHz}}{2}=35 \mathrm{kHz}$ In Abb. 1 ist der Versuchsaufbau dargestellt. Die Faden-

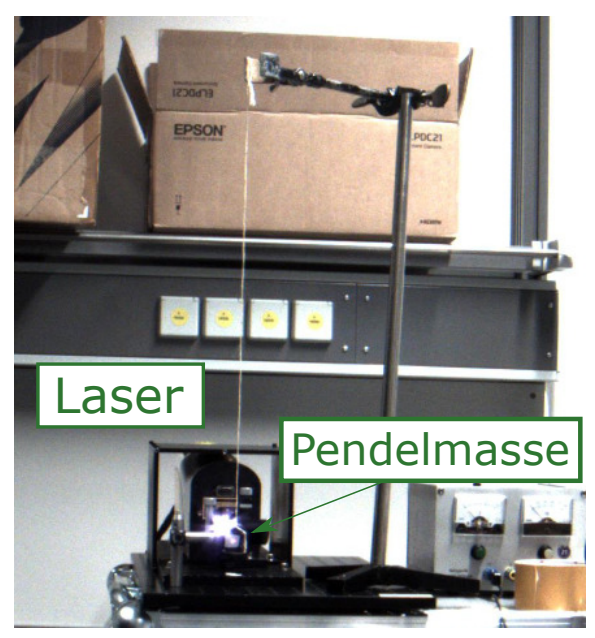

Abb. 1: Versuchsaufbau zur Kraftschätzung mit Pendelmethode.

länge des Pendels $l$ beträgt $0,5 \mathrm{~m}$. Die Eigenfrequenz des Pendelsystems ergibt sich mit der Erdbeschleunigung $g$ $\mathrm{zu} \omega_{0}=\sqrt{g / l} \approx 4,43 \mathrm{~Hz}$, so dass die untere Grenze des
Schätzbereichs bei ca. $2 \mathrm{~Hz}$ liegen sollte. Ein Nd:YAGPulslaser der Firma Quantel (Brilliant EaZy) mit einer Wellenlänge von $1064 \mathrm{~nm}$, einer Impulsdauer von etwa $5 \mathrm{~ns}$ und einem Durchmesser von $6 \mathrm{~mm}$ wird für den Versuch eingesetzt. Dieser Laser hat eine maximale Impulsenergie von $330 \mathrm{~mJ}$ und einen radialen Divergenzwinkel von 0,5 mrad. Direkt am Strahlausgang des Lasers wird ein Abschwächer platziert, um die Impulsenergie variieren zu können. Die minimale Leistungsdichte zur Erzeugung eines Plasmas liegt bei etwa $10^{11} \frac{\mathrm{W}}{\mathrm{cm}^{2}}$ [11]. Damit diese Voraussetzung erfüllt wird, wird der Laserstrahl noch mit Hilfe einer plankonvexen Linse (Thorlabs, LA4148 - YAG) fokussiert. Die Impulsenergie vor der Fokussierung wird mit einem Energiemeter (Thorlabs, ES220C) gemessen. Die eingestellte Impulsenergie beträgt in diesem Versuch etwa 240 mJ. Die Druckwellenfront nach der Plasmaentstehung trifft zunächst mittig auf die Vorderseite der Pendelmasse. Die Geschwindigkeit der Pendelmasse wird mittig auf ihrer Rückseite mit einem Laser-Doppler-Vibrometer (Polytec, MPV800) erfasst.

\section{Ergebnis}

In Abb. 2 ist der im Versuch gemessene Geschwindigkeitsverlauf bei laserinduzierter Anregung als durchgezogene Linie (in Grau) über der Messzeit dargestellt. Der Aus-

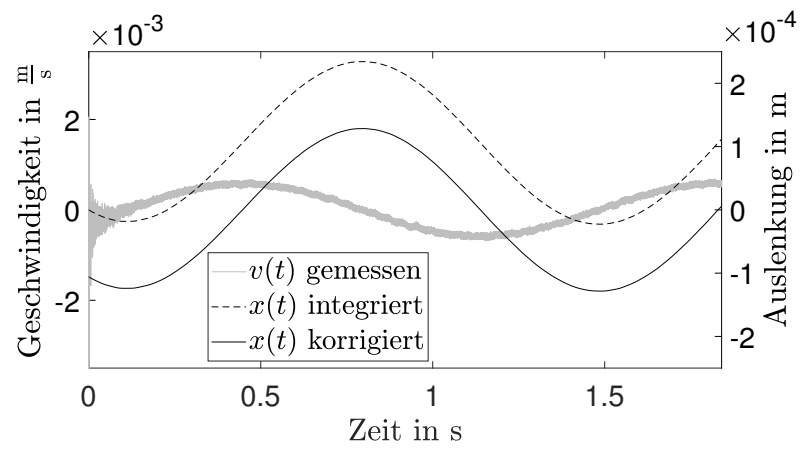

Abb. 2: Geschwindigkeits- und Auslenkungsverläufe.

lenkungsverlauf der Pendelmasse, der in Abb. 2 als gestrichelte Linie dargestellt ist, lässt sich näherungsweise durch numerische Integration der gemessenen Geschwindigkeit über der Zeit berechnen. Aus dem Auslenkungsverlauf kann die Auslenkungsamplitude des Pendels $\hat{x}$ schätzungsweise durch $\frac{x_{\max }-x_{\min }}{2}$ berechnet werden. Dabei sind $x_{\max }$ und $x_{\min }$ der maximale und minimale Wert im Auslenkungsverlauf. Die Auslenkungsamplitude beträgt in diesem Fall 1,284 $\cdot 10^{-4} \mathrm{~m}$. Allerdings darf 
der Schwingungsverlauf des Pendels keinen Offset aufweisen, weil die Auslenkung des Pendels in der Ruheposition $x_{\text {Ruhe }}=0 \mathrm{~m}$ sein muss. Der Auslenkungsverlauf wird daher um $-\frac{x_{\max }+x_{\min }}{2}=-1,058 \cdot 10^{-4} \mathrm{~m}$ verschoben. Der korrigierte Auslenkungsverlauf ist in Abb. 2 als durchgezogene Linie (in Schwarz) dargestellt. Aus der Amplitude ergibt sich, dass der maximale Auslenkungswinkel des Pendels $\varphi_{\max }$ weit unter $1^{\circ}$ liegt. Dies erlaubt die Vereinfachung $\varphi(t) \approx \sin \varphi(t)=\frac{x(t)}{l}$ mit der Auslenkung $x(t)$ und der Fadenlänge $l$.

Da die Anfangsposition der Pendelmasse $x_{0} \neq 0$ ist, ist es ersichtlich, dass diese Pendelbewegung zum großen Teil bereits vor dem Eintreffen der Stoßwelle vorhanden ist. Diese Pendelbewegung klingt nur sehr langsam ab und beeinflusst daher die Auswertung bei niedrigen Frequenzen, zumal die Pendelauslenkung zu Beginn der Messung variiert. Der Anregungskraftverlauf $f_{\mathrm{P}}(t)$ lässt sich, unter der Starrkörperannahme, durch das Aufstellen der Kräftebilanz an der Pendelmasse

$$
f_{\mathrm{P}}(t)-m g \sin \varphi(t)=m \frac{\mathrm{d} v(t)}{\mathrm{d} t}
$$

aus dem gemessenen Geschwindigkeitsverlauf $v(t)$ berechnen. Die Beschleunigung der Pendelmasse wird durch die resultierende Kraft aus Plasmaanregung und Fadenkraft hervorgerufen. In Abb. 3 wird der aus der Geschwindigkeitsmessung berechnete Anregungskraftverlauf $f_{\mathrm{P}}(t)$ über der Zeit von 0 bis $0,125 \mathrm{~ms}$ als gestrichelte Linie dargestellt. Der als durchgezogene Linie dargestellte Anregungskraftverlauf wird nach dem in Abschnitt 2.2 beschriebenen Simulationsmodell (vgl. Gl. (14)) berechnet. Es besteht

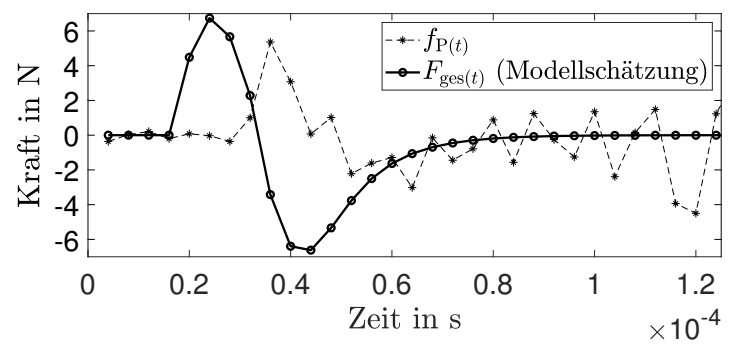

Abb. 3: Vergleich der Kraftverläufe durch Modellschätzung und Pendelmethode.

eine Zeitverschiebung von etwa $0,014 \mathrm{~ms}$ zwischen dem Anstieg der Kraft in beiden Kurven. Mögliche Ursachen dafür werden in Abschnitt 4 diskutiert. Der charakteristische Anregungsverlauf ist jedoch in beiden Kurven zu erkennen. Die Schwankungen nach dem ersten Kraftmaximum in der Kurve von $f_{\mathrm{P}}(t)$ können durch die Eigenschwingungen des Würfels (Pendelmasse) hervorgerufen werden. Dies lässt sich durch einen Vergleich zwischen den beiden Kurven nach einer Filterung mit einem linearphasigen FIR-Tiefpass (Grenzfrequenz $35 \mathrm{kHz}$ ) bestätigen (Abb. 4). Die hochfrequenten Komponenten stammen aus

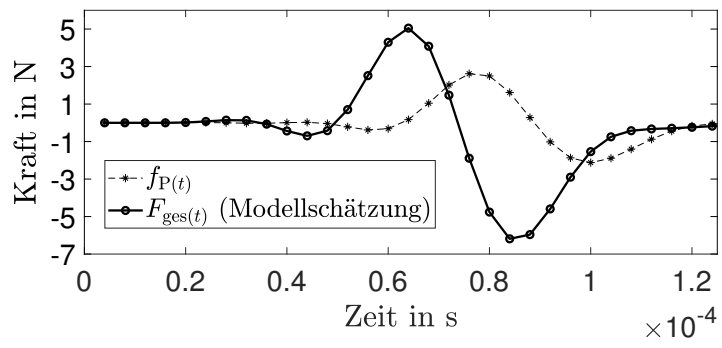

Abb. 4: Kraftvergleich (gefiltert bis $35 \mathrm{kHz}$ ).

dem Frequenzbereich, in dem der Einfluss durch die Eigenfrequenzen der Pendelmasse nicht vernachlässigbar ist. Der charakteristische Verlauf der Impulsanregung ist in beiden Kurven der Abb. 4 weiterhin zu erkennen.

In Abb. 5 sind die Amplitudendichtespektren der beiden Kraftverläufe von Abb. 4 dargestellt. Die Schätzung

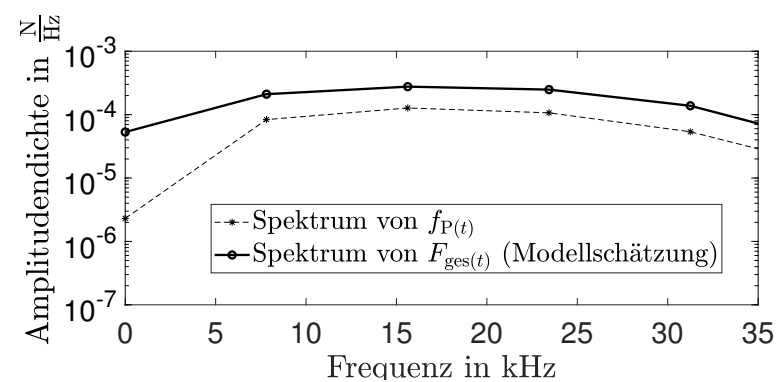

Abb. 5: Amplitudendichtespektrum der geschätzten Anregungskraft (Resolution-Bandwidth, RBW $=8 \mathrm{kHz}$, Abtastrate $\left.f_{\mathrm{S}}=250 \mathrm{kHz}\right)$.

des Simulationsmodells und der Messung werden als durchgezogene Linie und gestrichelte Linie dargestellt. Insgesamt stimmen beide Verläufe in Abb. 5 gut überein. Da für den unteren Frequenzbereich $(<8 \mathrm{kHz})$ zahlreiche schätzungsfreie direkte Kraftmesstechniken, wie z.B. Modalhammer, vorhanden sind, wird der Vergleich beider Schätzmethoden hier im Frequenzbereich von $8 \mathrm{kHz}$ bis $35 \mathrm{kHz}$ betrachtet. Die Schätzung durch das Simulationsmodell zeigt eine Überschätzung von $6 \mathrm{~dB}$ bis $8 \mathrm{~dB}$ in diesem Frequenzbereich. 


\section{Diskussion}

Mögliche Ursachen für die Zeitverschiebung des Kraftanstieges in Abb. 3 können Vereinfachungen und Ungenauigkeiten im Modell, Zeitverzögerungen durch Tiefpassfilter im Messsystem oder Verzögerungen durch das TriggerSignal sein. Allerdings wirkt sich ein reiner Zeitversatz bis auf eine zusätzliche lineare Phase nicht auf die Bestimmung der Übertragungsfunktion aus.

Eine weitere mögliche Ursache für die Zeitverschiebung in Abb. 3 ist die Unsicherheit des Abstands zwischen dem Druckursprung und der Testobjektoberfläche $d_{\mathrm{O}}$. Dieser Abstand wird aus dem Abstand zwischen der Fokuslinse und der Testobjektfläche unter Berücksichtigung der Brennweite berechnet. Da dieser Abstand mit einem Lineal abgemessen wird, kann die resultierende Berechnung hohe Unsicherheiten aufweisen. Bei der Messung in Abschnitt 3 wurde ein Abstand $d_{\mathrm{O}}$ von etwa $9,5 \mathrm{~mm}$ gemessen. Mit steigendem $d_{\mathrm{O}}$, d.h. größer werdendem $r$ in Gl. (12), verzögert sich der Zeitpunkt des Eintreffens der Wellenfront $t_{0, \text { ak }}$. Somit verzögert sich auch der durch das Modell geschätzte Kraftanstieg (vgl. Abb. 3).

In dem aktuellen Simulationsmodell wird eine vollständige Reflexion der Wellenfront beim Eintreffen der Welle auf die Oberfläche des Testobjekts angenommen. Somit wird die Modellschätzung mit einer Druckverdoppelung an der Gas-Festkörper-Grenzfläche berechnet (vgl. Gl. (13)). Dies kann ein Grund dafür sein, dass das Simulationsmodell ein höheres Kraftspektrum im Vergleich zu der Schätzung durch die Pendelmethode schätzt (vgl. Abb. 5).

\section{Zusammenfassung}

Unsere Bestimmung des Anregungsspektrums liefert ein vergleichbares Ergebnis wie die aus dem Stand der Wissenschaft bekannte Pendelmethode. Durch das Simulationsmodell kann der Einfluss von Änderungen der Testbedingungen, wie z.B. die Änderung der Laserenergie, schnell berechnet werden. Das breitbandige Anregungsspektrum ermöglicht die FRF-Bestimmung einer Struktur im höheren Frequenzbereich (über $20 \mathrm{kHz}$ ), welcher empfindlich gegen kleine Schädigungen in der Struktur ist. Aktuell werden Forschungen zur Laseranregung mit einem Mehrkanalvibrometer für die Schadenserkennung im Maschinenbetrieb durchgeführt, wobei bisher nur die gemessenen Antwortsignale ohne bekanntes Anregungsspektrum verwendet wurden [1]. Mit Hilfe des vorgestellten Berechnungsverfahrens können FRFs eines Testobjekts breitbandig durch das berechnete Anregungsspektrum bestimmt werden, wodurch eine effizientere Schadenserkennung möglich wird.

\section{Literatur}

[1] X. Cao und C. Rembe. Non-contact damage detection under operational conditions with multipoint laservibrometry. Sensors, 20(3):732, 2020.

[2] X. Cao, B. Tarigan, C. Rembe und H. Schwarze. Schadenserkennung an Wälzlagern. tm - Technisches Messen, 84(s1): 34-41, 2017.

[3] X. Cao, B. Tarigan, C. Rembe und H. Schwarze. Vibrationsbasierende white-etching-cracks-detektion an wälzlagern. tm - Technisches Messen, 85(6):443-453, 2018.

[4] D. J. Ewins. Modal Testing: Theory, Practice and Application. RESEARCH STUDIES PRESS LTD., Baldock, 2 Auflage, 2000.

[5] V. B. Georgiev, V. V. Krylov, Q. Qin und K. Attenborough. Generation of flexural waves in plates by laser-initiated airborne shock waves. Journal of Sound and Vibration, 330 (2):217 - 228, 2011.

[6] N. Hosoya, I. Kajiwara und T. Hosokawa. Vibration testing based on impulse response excited by pulsed-laser ablation: Measurement of frequency response function with detectionfree input. Journal of Sound and Vibration, 331(6):1355 1365, 2012.

[7] N. Hosoya, M. Nagata, I. Kajiwara und R. Umino. Nanosecond laser-induced plasma shock wave in air for noncontact vibration tests. Experimental Mechanics, 56(7):1305 - 1311, 2016.

[8] F. G. Kollmann, T. F. Schösser und R. Angert. Praktische Maschinenakustik. Springer, Berlin, 2 Auflage, 2006.

[9] R. Kowarsch und C. Rembe. Heterodyne interferometry at ultra-high frequencies with frequency-offset-locked semiconductor lasers. Measurement Science and Technology, 31(7): $075201,2020$.

[10] V. V. Krylov. On the theory of surface acoustic wave generation by electric spark discharge. Journal of Physics D: Applied Physics, 25(2):155, 1992.

[11] J. F. Ready. Industrial Applications of Lasers. Academic Press, San Diego, 2 Auflage, 1997.

[12] H. Schlichting. Grenzschicht-Theorie. Springer, Heidelberg, 10 Auflage, 2006.

[13] L. I. Sedov. Similarity and Dimensional Methods in Mechanics. Academic Press, New York, 1 Auflage, 1959.

[14] Y. B. Zel'dovivh und Y. P. Raizer. Physics of Shock Waves and High-Temperature Hydrodynamic Phenomena. Academic Press, New York, 1967. 